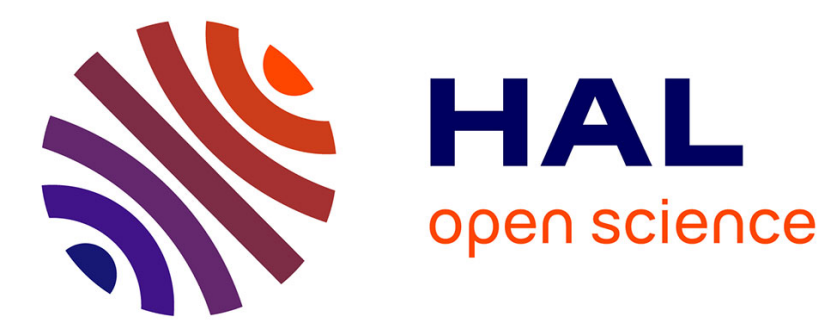

\title{
Introduction to the Special Issue on Breaking Waves
} Henrik Kalisch, Mario Ricchiuto, Philippe Bonneton, Mathieu Colin, Pierre Lubin

\section{To cite this version:}

Henrik Kalisch, Mario Ricchiuto, Philippe Bonneton, Mathieu Colin, Pierre Lubin. Introduction to the Special Issue on Breaking Waves. European Journal of Mechanics - B/Fluids, 2019, 73, pp.1-5. 10.1016/j.euromechflu.2018.11.007 . hal-02355203

\section{HAL Id: hal-02355203 https://inria.hal.science/hal-02355203}

Submitted on 8 Nov 2019

HAL is a multi-disciplinary open access archive for the deposit and dissemination of scientific research documents, whether they are published or not. The documents may come from teaching and research institutions in France or abroad, or from public or private research centers.
L'archive ouverte pluridisciplinaire HAL, est destinée au dépôt et à la diffusion de documents scientifiques de niveau recherche, publiés ou non, émanant des établissements d'enseignement et de recherche français ou étrangers, des laboratoires publics ou privés. 


\title{
Introduction to the Special Issue on Breaking Waves
}

\author{
Henrik Kalisch*, Mario Ricchiuto*, ${ }^{* *}$ Philippe Bonneton ${ }^{+}$, \\ Mathieu Colin**, and Pierre Lubin ${ }^{\$}$ \\ * Department of Mathematics, University of Bergen, \\ Postbox 7800, 5020 Bergen, Norway \\ ** Team CARDAMOM, Inria Bordeaux Sud-Ouest, \\ 200 av. de la vieille Tour, 33405 Talence cedex, France \\ + University of Bordeaux, CNRS, UMR 5805 EPOC, \\ Allée Geoffroy Saint-Hilaire, 33615 Pessac, France \\ $\S$ University of Bordeaux, Bordeaux-INP, I2M UMR CNRS 5295, \\ 16 avenue Pey-Berland, 33607 Pessac, France
}

This special issue comprises papers which were presented at the B'Waves workshops 2014 in Bordeaux, France and 2016 in Bergen, Norway. The B'Waves workshop series was conceived as a vehicle to facilitate further research into breaking of water waves through focussed week-long meetings featuring a limited number of speakers and with ample time allocated for discussions between presentations. Some new collaborations were initiated during and after B'Waves 2014, and some of the results contained in this special issue are based on these efforts. The third workshop in the series, B'Waves 2018 was held in Marseilles, France from May 28th to June 1 st.

One of the reasons for initiating this series of dedicated workshops was the observed inadequacy of the current state of knowledge about wave breaking. While wave breaking is one the most ubiquitous and well known phenomena in the worlds oceans and rivers, our current understanding of why, how and when it happens is still limited. While a fair amount is known today about possible pathways to breaking through instabilities and various types of forcing, the breaking process itself remains poorly understood due to its highly turbulent nature.

If you ask any person about wave breaking, the chance is very high that they have a clearly defined idea about what they understand to be wave breaking, with most people probably imagining barrel waves at some of the worlds most famous beaches. Other types of wave breaking which are less spectacular to the casual observer and maybe less well known but equally important are wave breaking in wave groups in the open ocean, white-capping, microbreaking and also breaking of internal waves. Over the years, a number of review articles and monographs have been published on the subject $[1,8,27,48,57,64,66,68]$, and these accounts invariably call for more research into nearly every aspect of wave breaking.

Even though wave breaking happens on a small scale and involves fluid motion and pressure gradients changing on a microscale, it has a major impact on the global energy balance in the world's oceans. Indeed, in fully developed seas, wave breaking is thought to be responsible for as much as $80 \%$ of energy dissipation [1]. This link between very small and global scales makes wave breaking a challenging multi-scale problem. 
As wave breaking plays an important role in the wave dynamics in the oceans, it has also been reviewed in accounts of wave forecasting such as [19] and others. Indeed, wave breaking has a large impact on a host of oceanic and coastal processes such as energy dissipation, current and vorticity creation, Lagrangian transport, mixing of the upper ocean and air-sea interaction $[11,23,45,58,72,71]$.

While the present introduction and special issue is mostly concerned with wave breaking at the surface of the ocean, it should be mentioned that breaking of internal waves is also an important phenomenon which is connected to global ocean circulation. Indeed, breaking of internal waves is partially responsible for maintaining the stable density stratification of the world oceans, and in particular, internal wave breaking is thought to be a major factor in the thermohaline circulation driving the exchange of warm and arctic waters [87].

Returning to surface waves, it appears that wave breaking usually happens on a very fast time scale, lasting only a fraction of a wave period [12, 73]. This is very short especially when compared to the time scale of wind driven waves which grow through wind forcing and nonlinear interactions over hundreds of kilometer and several hours and days [42, 49]. While all ocean waves continuously receive energy from the wind via air-sea interaction, it was estimated in [3] that only about $10 \%$ of waves break unless wind forcing is very strong. Nevertheless, wave breaking provides a very efficient energy dissipation mechanism. Indeed it was shown for example in [35] that wave breaking features high turbulence levels with dissipation rates up to four orders of magnitude larger than the average background values in the ocean.

The intermittency and random nature of wave breaking makes it difficult to study in the laboratory, and even more difficult to detect in the field. Indeed, the study of wave breaking often incorporates some element of a visual analysis such as for example the combined visual and data analysis of [39]. Using white-capping as an indicator for breaking can also be tricky since after a wave has broken, white-capping may persist for a few more wave periods, complicating even a visual analysis [73]. Given the many factors influencing the occurrence, and nature of wave breaking and its highly nonlinear and turbulent character, it is clear that a complete understanding of the breaking process and its many causes and implications is out of reach at this point.

One of the open issues surrounding wave breaking is its very definition: There is no clear single criterion that signals the commencement of wave breaking, though most researcher would probably agree with the following statement by the authors of [8]: "It is generally recognized that an individual wave breaking event usually starts when water particles near a wave crest develop a velocity in the wave propagation direction sufficiently large for them to fall down the front of the wave".

Partially as a result of this lack of a clearly defined indicator of wave breaking the detection of wave breaking through various wave breaking criteria has received a lot of attention. As delineated in $[81,86]$, there are essentially three classes of breaking criteria. Geometric criteria are based on the shape and in particular the steepness of the waves close to breaking, such as reviewed in [2]. Kinematic criteria are based on violation of the kinematic free surface condition, the most well known criterion being if the particle velocity exceeds the crest velocity, or in other words if a stagnation point appears on the free surface at the crest, such as in Stokes's highest wave.

The kinematic criterion has traditionally been used in conjunction with the phase velocity or group velocity $[76,86]$, but recent studies have also focused on the crest velocity. In some cases, evidence pointed to the necessity of tightening of the criterion [5] while in other cases, the kinematic criterion has been verified fairly closely [40]. The criterion has also been studied 
in the context of internal waves [37].

Dynamic criteria are based on accelerations exceeding some multiple of the gravitational acceleration [69]. A subclass of the dynamic criteria are the energetic criteria based on an evaluation of energy flux and density, such as put forward in $[9,75]$, There is a host of studies investigating these various criteria, and each one of them has been validated in at least some cases $[2,9,5,40,81,76,86]$. While some authors continue the quest for a single criterion fitting all cases, it also reasonable to surmise that some criteria may work only in certain cases given the large variety of situations in which breaking may occur.

Water waves breaking on beaches can be classified into a range of different types [34]. The most common types of breaking are spilling, plunging, collapsing and surging, each appearing in a certain parameter regime depending on waveheight, initial depth and bottom slope in an idealized setting [36]. In deep water, wave breaking can be roughly classed into plunging, collapsing and microbreaking. Microbreaking is especially difficult to detect as it is not accompanied by whitecapping [84].

The evolution of the wave prior to the breaking point may vary significantly from case to case. In many cases, waves break because of some forcing be it wind forcing [3], wave-current interaction [74], bottom forcing in coastal regions [98, 16] or a forced discharge [30]. As the wave approaches the breaking point, capillary effects invariably come to the fore, as shown for example in [31] and the photographs depicted in [28],

In some cases, waves break as a result of intrinsic hydrodynamic processes. For example it is well known by now that various instabilities may lead to wave breaking. The dynamics leading to wave breaking due to such intrinsic instabilities are fairly well understood. In particular, it is clear that modulational instabilities may lead to wave breaking [55] and the instability featured in $[79,80]$ and other types of crest instabilities also lead to wave breaking $[46,56]$. Three-dimensional instabilities have also been studied intensively [15, 62, 63, 32] as have the appearance of three-dimensional structures prior to breaking [4] and after breaking [59, 58, 33].

One issue of importance to the approximate numerical description of oceanic and coastal dynamics is the incorporation of wave breaking into simulation tools. For coastal areas, the traditional method is the inclusion of surface rollers such as advocated in $[61,78]$ or using a localized eddy viscosity $[52,50]$. If hyperbolic equations of shallow-water type are used, then the energy-dissipative property of shock solutions can be used to dissipate energy lost due to breaking waves This method has been refined lately in a number of papers $[13,17,22,47$, 83]. The intermittency of the wave breaking process can be simulated by switching between dispersive and hyperbolic models with the help of some numerical wave breaking criterion $[14,5]$.

For ocean waves, the dissipation due to breaking waves is parameterized into the spectral energy equation essentially using ideas of $[54,70,89,26]$ and others. While various approaches exist (see for example the weak turbulence model explained in [90]), the white-capping models of [43] seems to be the most widely used model for breaking mechanism in wave-forecast models.

Many of the works mentioned above are classical by now as scientific research aimed at an understanding of wave breaking has spanned many decades. Due to the many open questions remaining, wave breaking is still a very active area of research, with a large number of groups using a variety of methods for their investigations. Among the innumerable laboratory studies and field campaigns, we mention $[20,41,25,29,51,65,77,85]$. Numerical simulations have been carried out using a variety of methods including multi-phase methods [59], levelset methods [44], volume of fluid (VOF) methods [53] large-eddy-simulations [21, 59], direct numerical simulations [38, 88], Reynolds-averaged Navier-Stokes equations [60, 91] and many others. 
Many more excellent works exist, and we refer the reader to the references in the articles included in this special issue. In the following we briefly introduce these contributions.

\section{Contributions in this issue:}

In The turbulent airflow over wind generated surface waves [101], Buckley and Véron present an experimental study of the turbulent structure of the airflow above wind generated surface waves. The turbulent boundary layer in the air phase is characterized by numerous velocity sweeps and ejections, accompanied by intense downwind-tilted spanwise shear layers originating from the surface. The authors are able to estimate the statistical significance of thesse turbulent events using quadrant analysis. It is shown that these events become phase-locked in the presence of waves.

The contribution by Filippini, Arpaia, Bonneton and Ricchiuto [97] contains a study of the formation of river bores detailing the numerical aspects behing the physical results discussed in [14]. The authors define certain parameters based on the estuary geometry the water depth, the amplitude and the angular frequency of the tidal wave. The authors study numerically the estuarine parameter space in order to identify physical conditions that lead to tidal bore generation. According to the result presented in this paper, bore formation is controlled by two competing physical processes: the distortion of the tidal wave, with flood dominance and eventually bore inception and the dissipation of the tidal wave which is unfavorable to bore formation.

The paper by Gavrilyuk, Liapidevskii and Chesnokov [96] is concerned with a two-layer model describing the interaction of a shear bubble layer formed by breaking waves and an underlying potential layer in the shallow-water approximation. A non-hydrostatic formulation taking into account the entrainment effects in shear flows is proposed. Time and space periodic solutions are found, and some basic problems concerning the formation of bores and periodic structures from a uniform flow are numerically approximated.

In the paper by Hur [94], the nonlinear shallow water equations are written in the presence of a background shear flow. Using Riemann invariants, the equations are restricted to one-way propagation, and dispersive terms are added to find the Korteweg-de Vries equation and the fully dispersive Whitham equation over shear flow. The equations are investigated with respect to derivative blow-up which means that the solution remains bounded but its slope becomes unbounded in finite time. It is shown that the solution of the vorticity-modified shallow water equations breaks down if it carries an increase of elevation; the breaking time decreases to zero as the size of vorticity increases.

The author also proposes a full-dispersion shallow water model, combining the dispersion relation of water waves and the nonlinear shallow water equations in the constant vorticity setting. It is shown that small-amplitude and periodic traveling waves are unstable to long wavelength perturbations if the wave number is greater than a critical value, and stable otherwise, similarly to the Benjamin-Feir instability in the irrotational setting. It is also shown that vorticity considerably alters the modulational stability and instability in the presence of surface tension.

In Effects of the wind on the breaking of modulated wave trains [100], Iafrati, De Vita and Verzicco study the effect of wind on the wave breaking induced by the modulational instability using the open source software Gerris. The two-phase flow is modelled by the two-dimensional 
Navier-Stokes equations for a single incompressible fluid and a Volume of Fluid technique is employed to capture the air-water interface. The simulation covers the initial development of the wind profile, the growth of the modulational instability, the breaking and post breaking phases. Results show the occurrence of air flow separation from the wave crests shortly after the initial start. It is shown that due to the flow separation there is a favourable pressure gradient about the wave crests whereas the tangential stresses are generally in favour of the wave propagation on the back of the wave but are opposed to the propagation along the forward face and in the wave trough. In agreement with the experiments, the growth rate of the side-bands is reduced when compared to the corresponding solution without wind.

In Solitary wave impact on a vertical wall [105] Jensen investigates wave impact on a vertical wall using both a wave tank and a numerical code. Two different impact phenomena are explored. A flip-through, where the front face of the wave moves rapidly vertically due to a jet and a case with a very steep wave. In the latter, the front of the wave is almost vertical, and the pressure at impact was 60 percent higher than the flip-through event. Measurements are compared with numerical simulations, and good agreement is found. In particular, maximum pressure excursion is found when a vertical front is violently impacting on a vertical wall. Interestingly, a pronounced double pressure peak is observed due to the impact and the rundown process.

In the paper by Kazolea, Filippini, Ricchiuto, Abadie, Medina, Morichon, Journeau, Marcer, Pons, Le Roy, Pedreros and Rousseau [92], benchmarks for various stages of a tsunami event are studied. The authors consider generation, propagation, run-up and inundation using different numerical codes, including models based on depth-averaged Boussinesq equations and a fully three-dimensional Navier-Stokes solver. The codes are used for a flow featuring propagation, run-up, overtopping and reflection, and compared with experimental data produced from a set of laboratory experiments carried out at the O.H. Hinsdale Wave Research Laboratory, Oregon State University. The authors conclude that from an operational point of view, the Boussinesq codes can be run more efficiently, and provide a similar level of accuracy as the code based on the full Navier-Stokes equations. Nevertheless, the Navier-Stokes solver is able to provide more detailed information on finer scales which may be required in the study of air entrainment and wave-structure interaction.

In the paper by Kirby and Derakhti [98], the effect of local variations of wave energy dissipation as a generation mechanism for vorticity is discussed. The authors use an LES/VOF model to examine the evolution of coherent vortex structures, considering a range of vorticitygenerating mechanisms ranging from the generation of patterns of vertical vorticity in $2 \mathrm{D}$, depth-averaged flows, to a more detailed picture of the vorticity field evolving during a localized breaking event. They study the persistence of three-dimensionality of these structures and their contribution to the development of depth-integrated vertical vorticity.

In Transient wave resistance upon a real shear current [95], Li, Smeltzer, and Ellingsen study the influence of background shear currents on the development of ship waves. In particular, the authors look at real-world data from the Columbia River in the northwestern United States, and model ships with dimensions and Froude numbers typical of three classes of vessels operating in these waters. The authors find that for smaller ships, the wave resistance can differ drastically from that in quiescent water, and depends strongly on Froude number and direction of motion.

In the paper Effect of Leading Waves on Velocity Distribution of Undular Bore Traveling 
over Sloping Bottom [103], Lin, Kao, Wong, Shao, Fu, Yuan and Raikar provide new insights into the effect of the leading wave on the velocity distribution of an undular bore. The complete evolution of the free surface elevation of the bore is measured at various locations. Based on visualized images and measurements of the velocity field key features of the bore are explored. In particular, the spatial and temporal variations of the flow field are investigated. The influence of the leading waves on flow acceleration behind the leading front is elucidated. The flow reversal zone is studied in detail, and shear layer formation near the sloping bottom is found. The development of a vortex train in the shear layer near the flow-reversal zone is observed.

In the contribution by Lubin, Kimmoun, Véron and Glockner [104], the authors study a varitey of instabilities, such as Rayleigh-Taylor, Kelvin-Helmholtz and other instabilitues which are responsible for features such as vortex formation, air-entrainment and droplet generation in breaking waves. Using numerical simulations and new experimental visualizations, the authods discuss the successive steps of atomization of a plunging liquid jet connected to a breaking wave.

The contribution of Lucarelli, Lugni, Falchi, Felli and Brocchini [93] focusses on understanding the internal flow structure in a sloshing-induced, rapidly-evolving spilling breaker. The article features an in-depth description of experiments conducted at the Sloshing Laboratory of the CNR-INSEAN (Marine Technology Research Institute), Rome, Italy, and a comparison with a simplified analytical model based on a three-layer structure of an underlying potential flow, a thin, turbulent single-phase layer in the center, and a turbulent two-phase layer near the free surface. The authors are able to describe in detail the development of a boundary layer near the free surface, and the development of vorticity and turbulent flow.

In the paper by Senthilkumar and Kalisch [99], the critical breaking waveheight for long surface water waves on a flow with constant vorticity in the KdV approximation is found. Given a background linear shear flow, a KdV equation can be found with coefficients depending on the strength of the shear flow. The derivation also shows that the velocity field under the wave can be constructed approximately from the free surface excursion.

A convective breaking criterion is put forward and used to detect incipient wave breaking in periodic traveling waves and solitary waves. As in the case of vanishing vorticity [10, 18], it is shown that for both the solitary wave and the cnoidal waves, there are limiting waveheights where the horizontal component of the particle velocity equals the phase velocity of the wave. It is found that the strength of the vorticity has a considerable influence on the critical waveheight.

InX-ray measurements of plunging breaking solitary waves, Smith, Kolaas, Jensen and Sveen examine the characteristics of air cavities generated by breaking solitary waves, utilizing a novel tomographic X-ray system. Small scale experiments of solitary waves that propagate on a (1:10) beach are conducted, and images are captured at locations from the surf zone to the swash zone and at maximum runup. A large air tube is observed right after the plunger impacts the dry beach, and the void velocity and shape of the large air tube are measured. The large air tube evolves from a symmetrical shape to an asymmetrical shape. Contrast enhanced X-ray images reveal that the swash tongue surface is unstable, and that secondary mixing of air and water occurs. X-ray images from the maximum runup reveal that the air is still entrapped by the thin swash tongue at times close to maximum runup [102]. 
Acknowledgments. We are grateful to F. Dias and G.J.F. van Heijst, the editors-in-chief of this journal for providing a forum for this collection of papers, and we thank the journal staff for providing support throughout the preparation of this Special Issue. Finally, we would like to thank the authors for submitting high-quality contributions, and the reviewers for their valuable service.

\section{References}

[1] Babanin, A., 2011. Breaking and dissipation of ocean surface waves. Cambridge University Press.

[2] Babanin, A., Chalikov, D., Young, I. and Savelyev, I., 2007. Predicting the breaking onset of surface water waves, Geophys. Res. Lett, 34, p.L07605.

[3] Babanin, A.V., Young, I.R. and Banner, M.L., 2001. Breaking probabilities for dominant surface waves on water of finite constant depth, Journal of Geophysical Research: Oceans, 106(C6), pp.11659-11676.

[4] Babanin, A.V., Waseda, T., Kinoshita, T. and Toffoli, A., 2011. Wave breaking in directional fields, Journal of Physical Oceanography, 41 (1), pp.145-156.

[5] P. Bacigaluppi, M. Ricchiuto and P. Bonneton, Upwind stabilized finite element modelling of nonhydrostatic wave breaking and run-up, hal-00990002 (2014).

[6] Banner, M.L., Barthelemy, X., Fedele, F., Allis, M., Benetazzo, A., Dias, F. and Peirson, W.L., 2014. Linking reduced breaking crest speeds to unsteady nonlinear water wave group behavior Physical review letters, 112 (11), p.114502.

[7] Banner, M.L. and Peirson, W.L., 1998. Tangential stress beneath wind-driven air-water interfaces, Journal of Fluid Mechanics, 364, pp.115-145.

[8] Banner, M.L. and Peregrine, D.H., 1993. Wave breaking in deep water, Annual Review of Fluid Mechanics, 25(1), pp.373-397.

[9] X. Barthelemy, M.L., Banner, W. L., Peirson, F., Fedele, M., Allis and F. Dias, On a unified breaking onset threshold for gravity waves in deep and intermediate depth water, J. Fluid Mech. in press (2018).

[10] M. Bjørkavåg and H. Kalisch, Wave breaking in Boussinesq models for undular bores, Phys. Lett. A 375 (2011), 1570-1578.

[11] Blenkinsopp, C.E. and Chaplin, J.R., 2007, Void fraction measurements in breaking waves Proceedings of the Royal Society of London A 463, 3151-3170.

[12] Bonmarin, P., 1989. Geometric properties of deep-water breaking waves, Journal of Fluid Mechanics, 209, 405-433.

[13] Bonneton, P., 2007. Modelling of periodic wave transformation in the inner surf zone, Ocean Engineering, 34(10), pp.1459-1471.

[14] P. Bonneton, A.G. Filippini, L. Arpaia, N. Bonneton and M. Ricchiuto, Conditions for tidal bore formation in convergent alluvial estuaries, Estuarine, Coastal and Shelf Science, doi:10.1016/j.ecss.2016.01.019.

[15] T.J. Bridges Wave breaking and the surface velocity field for three-dimensional water waves, Nonlinearity 22 (2009), 947-953.

[16] Briganti, R., Musumeci, R.E., Bellotti, G., Brocchini, M. and Foti, E. Boussinesq modelling of breaking waves: description of turbulence J. Geophys. Res. Oceans 109, 2004, 7015.

[17] Brocchini, M. and Dodd, N., 2008. Nonlinear shallow water equation modeling for coastal engineering, Journal of waterway, port, coastal, and ocean engineering, 134(2), pp.104-120.

[18] M.K. Brun and H. Kalisch, Convective wave breaking in the KdV equation, Anal. Math. Phys. 8 (2018), 57-75. 
[19] Cavaleri, L., Alves, J.H., Ardhuin, F., Babanin, A., Banner, M., Belibassakis, K., Benoit, M., Donelan, M., Groeneweg, J., Herbers, T.H.C. and Hwang, P.A.E.M., 2007. Wave modelling-the state of the art. Progress in oceanography, 75(4), pp.603-674.

[20] K.-A. Chang and Liu, P.F.L. 1998 Velocity, acceleration and vorticity under a breaking wave, Phys. Fluids 10, 327-329.

[21] Christensen, E.D. and Deigaard, R., 2001. Large eddy simulation of breaking waves, Coastal engineering, 42, 53-86.

[22] Cienfuegos, R., Barthélemy, E. and Bonneton, P., 2009. Wave-breaking model for Boussinesq-type equations including roller effects in the mass conservation equation, Journal of waterway, port, coastal, and ocean engineering, 136(1), pp.10-26.

[23] Deike, L., Pizzo, N., and Melville, W. (2017). Lagrangian transport by breaking surface waves, Journal of Fluid Mechanics, 829, 364-391.

[24] Deike, L., Popinet, S., and Melville, W. (2015). Capillary effects on wave breaking, Journal of Fluid Mechanics, 769, 541-569.

[25] J.D. Diorio, X. Liu and J.H. Duncan, An experimental investigation of incipient spilling breakers, J. Fluid Mech. 633 (2009), 271-283.

[26] Donelan, M.A. and Pierson, W.J., 1987. Radar scattering and equilibrium ranges in wind-generated waves with application to scatterometry, Journal of Geophysical Research: Oceans, 92(C5), pp.4971-5029.

[27] J.H. Duncan, Spilling breakers Ann. Rev. Fluid Mech. 33 (2001), 519-547.

[28] J.H. Duncan, V. Philomin, M. Behres and J. Kimmel, The formation of a spilling breaker, Phys. Fluids. 6 (1994), S2.

[29] J.H. Duncan, H. Qiao, V. Philomin and A. Wenz, Gentle spilling breakers: crest profile evolution, J. Fluid Mech. 379 (1999), 191-222.

[30] H. Favre, Ondes de Translation, (Dunod, Paris, 1935).

[31] Fedorov, A.V. Melville, W.K. 1998 Nonlinear gravity-capillary waves with forcing and dissipation Journal of Fluid Mechanics 354, 1-42

[32] M. Francius and C. Kharif, Three-dimensional instabilities of periodic gravity waves in shallow water, J. Fluid Mech. 561 (2006), 417-437.

[33] O.B. Fringer and R.L. Street, The dynamics of breaking progressive interfacial waves, J. Fluid Mech. 494 (2003), 319-353.

[34] C.J. Galvin, Breaker type classification on three laboratory beaches, J. Geoph. Res. 73 (1968), $3651-3659$.

[35] J.R. Gemmrich and D.M. Farmer, Near-surface turbulence in the presence of breaking waves, J. Phys. Oceanography, 34 (2004), 1067-1086.

[36] S.T. Grilli, I.A. Svendsen, and R. Subramanya, Breaking criterion and characteristics for solitary waves on slopes, J. Waterways Port Coastal Ocean Engng. 123 (1997), 102-112.

[37] J. Grue, A., Jensen, P.O.., Rusås and J.K. Sveen, J.K., 1999. Properties of large-amplitude internal waves, J. Fluid Mech. 380, 257-278.

[38] P. Guyenne and S. Grilli, Numerical study of three-dimensional overturning waves in shallow water, J. Fluid Mech. 547 (2006) 361-388.

[39] Holthuijsen, L.H. and Herbers, T.H.C., 1986. Statistics of breaking waves observed as whitecaps in the open sea. Journal of Physical Oceanography, 16(2), pp.290-297.

[40] U. Itay and D. Liberzon, Lagrangian kinematic criterion for the breaking of shoaling waves, J. Phys. Ocean. 47 (2017), 827-833.

[41] A. Jensen, G.K. Pedersen and D.J. Wood, An experimental study of wave run-up at a steep beach, J. Fluid Mech. 486 (2003), 161-188. 
[42] Hasselmann, K., 1962. On the non-linear energy transfer in a gravity-wave spectrum Part 1. General theory, Journal of Fluid Mechanics, 12(4), pp.481-500.

[43] Hasselmann, K., 1974. On the spectral dissipation of ocean waves due to white capping, BoundaryLayer Meteorology, 6(1-2), pp.107-127.

[44] Iafrati, A., 2009. Numerical study of the effects of the breaking intensity on wave breaking flows, Journal of Fluid Mechanics, 622, 371-411.

[45] Iafrati A (2011) Energy dissipation mechanisms in wave breaking processes: spilling and highly aerated plunging breaking events, J Geophys Res 116, C07024.

[46] Jillians, W.J., 1989. The superharmonic instability of Stokes waves in deep water, Journal of Fluid Mechanics, 204, pp.563-579.

[47] M. Kazolea, A. Delis and C. Synolakis, Numerical treatment of wave breaking on unstructured finite volume approximations for extended Boussinesq-type equations, J. Comput. Phys. (2014).

[48] Kiger KT, Duncan JH. 2012. Air entrainment mechanisms in plunging jets and breaking waves, Annu. Rev. Fluid Mech. 44, 563-596.

[49] B. Kinsman, Wind Waves (Prentice-Hall, Englewood Cliffs, 1965).

[50] Kirby, J.T., Wei, G., Chen, Q., Kennedy, A.B. and Dalrymple, R.A., 1998. FUNWAVE 1.0: fully nonlinear Boussinesq wave model-Documentation and user's manual, research report NO. CACR98-06.

[51] Kimmoun O, Branger H (2007) A particle image velocimetry investigation on laboratory surf-zone breaking waves over a sloping beach. J Fluid Mech 588, 353-397.

[52] Kennedy, A.B., Chen, Q., Kirby, J.T. and Dalrymple, R.A., 2000. Boussinesq modeling of wave transformation, breaking, and runup. I: 1D. Journal of waterway, port, coastal, and ocean engineering, 126(1), pp.39-47.

[53] Lin, P. and Liu, P.L.F., 1998. A numerical study of breaking waves in the surf zone, Journal of Fluid Mechanics, 359, 239-264.

[54] Longuet-Higgins, M.S., 1969. On wave breaking and the equilibrium spectrum of wind-generated waves. Proc. Roy. Soc. London A, 310, pp.151-159.

[55] Longuet-Higgins, M.S. and Cokelet, E.D., 1978. The deformation of steep surface waves on water ll. Growth of normal-mode instabilities, Proc. R. Soc. Lond. A, 364(1716), pp.1-28.

[56] M.S. Longuet-Higgins and D.G. Dommermuth, Crest instabilities of gravity waves. Part 3. Nonlinear development and breaking, J. Fluid Mech. 336 (1997), 33-50.

[57] Lubin, P. and Chanson, H., 2017. Are breaking waves, bores, surges and jumps the same flow? Environmental Fluid Mechanics, 17 (1), pp.47-77.

[58] Lubin, P. and Glockner, S., 2015. Numerical simulations of three-dimensional plunging breaking waves: generation and evolution of aerated vortex filaments, Journal of Fluid Mechanics, 767, pp.364-393.

[59] Lubin, P., Vincent, S., Abadie, S. and Caltagirone, J.P. Three-dimensional large eddy simulation of air entrainment under plunging breaking waves, Coast. Engrng, 53 (2006), 631-655.

[60] Ma G., Shi F., J.T. Kirby, Shock-capturing non-hydrostatic model for fully dispersive surface wave processes, Ocean Modell., 43 (2012), 22-35

[61] Schäffer, H.A., Madsen, P.A. and Deigaard, R., 1993, A Boussinesq model for waves breaking in shallow water, Coastal Engineering, 20, 185-202.

[62] J.W. McLean, Instabilities of finite amplitude gravity waves on water of finite depth, J. Fluid Mech 114 (1982), 331-341.

[63] Melville, W.K., 1982. The instability and breaking of deep-water waves, Journal of Fluid Mechanics, 115, pp.165-185. 
[64] Melville, W.K., 1996. The role of surface-wave breaking in air-sea interaction, Annual review of fluid mechanics, 28(1), pp.279-321.

[65] K. Melville, F. Véron and C.J. White, The velocity field under breaking waves: coherent structures and turbulence, J. Fluid Mech. 454 (2002), 203-233.

[66] Peregrine, D.H., 1983. Breaking waves on beaches, Annual Review of Fluid Mechanics, 15(1), pp.149-178.

[67] Perlin M, Schultz WW. 2000. Capillary effects on surface waves, Annu. Rev. Fluid Mech. 32, 241-274.

[68] M. Perlin, W. Choi, and Z. Tian, Breaking waves in deep an intermediate waters, Ann. Rev. Fluid Mech. 45 (2013), 115-145.

[69] Phillips, O.M., 1958. The equilibrium range in the spectrum of wind-generated waves. Journal of Fluid Mechanics, 4( 4), pp.426-434.

[70] Phillips, O.M., 1985. Spectral and statistical properties of the equilibrium range in wind-generated gravity waves, Journal of Fluid Mechanics, 156, pp.505-531.

[71] Pizzo, N., Deike, L., and Melville, W. (2016). Current generation by deep-water breaking waves, Journal of Fluid Mechanics, 803, 275-291.

[72] Pizzo, N.E. and Melville, W.K. (2013). Vortex generation by deep-water breaking waves, Journal of Fluid Mechanics, 734, 198-218.

[73] Rapp, R.J. and Melville, W.K., 1990. Laboratory measurements of deep-water breaking waves, Phil. Trans. R. Soc. Lond. A, 331 (1622), pp.735-800.

[74] Romero, L., Lenain, L. and Melville, W.K., 2017. Observations of Surface Wave-Current Interaction, Journal of Physical Oceanography, 47, 615-632.

[75] J.B. Song and M.L. Banner, On determining the onset and strength of breaking for deep water waves. Part I: Unforced irrotational wave groups, J. Physical Oceanography 32 (2002), 2541-2558.

[76] P. Stansell and C. MacFarlane, Experimental investigation of wave breaking criteria based on wave phase speeds, J. Phys. Oceanography 32 (2002), 1269-1283.

[77] Sutherland, P. and Melville, W.K., 2015. Field measurements of surface and near-surface turbulence in the presence of breaking waves, Journal of Physical Oceanography, 45 943-965.

[78] Svendsen, I.A., 1984. Wave heights and set-up in a surf zone, Coastal engineering, 8 (4), pp.303329.

[79] M. Tanaka, 1983 The stability of steep gravity waves, J. Phys: Soc. Japan 52, 3047-3055.

[80] M. Tanaka, J.W. Dold, M. Lewy and D.H. Peregrine, Instability and breaking of a solitary wave, J. Fluid Mech. 185 (1987) 235-248.

[81] Z. Tian, M. Perlin and W. Choi, Evaluation of a deep-water wave breaking criterion, Phys. Fluids 20 (2008), 066604.

[82] M. Tissier, P. Bonneton, F. Marche, F. Chazel, and D. Lannes, A new approach to handle wave breaking in fully non-linear Boussinesq models, Coast. Engineering 67 (2012), 54-66.

[83] M. Tonelli and M. Petti, Simulation of wave breaking over complex bathymetries by a Boussinesq model, J. Hydr. Research 49 (2011), 473-486.

[84] Tulin, M.P. and Landrini, M., 2001. Breaking waves in the ocean and around ships, in Twenty-Third Symposium on Naval Hydrodynamics, Office of Naval Research Bassin d'Essais des CarenesNational Research Council.

[85] C.P. Tsai et al. Examination of empirical formulas for wave shoaling and breaking on steep slopes, Ocean Eng. 32 (2005), 469-483.

[86] C.H. Wu and H.M. Nepf, Breaking criteria and energy losses for three-dimensional wave breaking J. Geophys. Res.: Oceans 107 (2002), C10. 
[87] C. Wunsch and R. Ferrari, Vertical mixing, energy, and the general circulation of the oceans, Annual Review of Fluid Mechanics 36 (2004), 281-314,

[88] Zh. Xue, A two-phase flow model for three-dimensional breaking waves over complex topography, Proc. Roy. Soc. London A 471 (2015), (20 pages).

[89] Yuan, Y., Tung, C.C. and Huang, N.E., 1986. Statistical characteristics of breaking waves, In Wave dynamics and radio probing of the ocean surface (pp. 265-272). Springer, Boston, MA.

[90] Zakharov, V.E., Korotkevich, A.O., Pushkarev, A. and Resio, D., 2007. Coexistence of weak and strong wave turbulence in a swell propagation, Physical review letters, 99(16), p.164501.

[91] M. Zijlema, G. Stelling, P. Smit, SWASH: an operational public domain code for simulating wave fields and rapidly varied flows in coastal waters, Coastal Eng., 58 (2011), 992-1012.

[92] M. Kazolea, A. Filippini, M. Ricchiuto, S. Abadie, M.M. Medina, D. Morichon, C. Journeau, R. Marcer, K. Pons, S. LeRoy and R. Pedreros, Wave propagation, breaking, and overtopping on a $2 D$ reef: A comparative evaluation of numerical codes for tsunami modelling, Eur. J. Mech. B Fluids, https://doi.org/10.1016/j.euromechflu.2017.10.010.

[93] A. Lucarelli, C. Lugni, M. Falchi, M. Felli, and M. Brocchini, On a layer model for spilling breakers: A preliminary experimental analysis, Eur. J. Mech. B Fluids, https://doi.org/10.1016/j.euromechflu.2017.07.003.

[94] V.M. Hur, Shallow water models with constant vorticity, Eur. J. Mech. B Fluids, https://doi.org/10.1016/j.euromechflu.2017.06.001.

[95] Y. Li, B.K. Smeltzer, and S. Å. Ellingsen, Transient wave resistance upon a real shear current, Eur. J. Mech. B Fluids, https://doi.org/10.1016/j.euromechflu.2017.08.012.

[96] S. Gavrilyuk, V., Liapidevskii, A. and Chesnokov, A., 2017. Interaction of a subsurface bubble layer with long internal waves, Eur. J. Mech. B Fluids, https://doi.org/10.1016/j.euromechflu.2017.07.004.

[97] A.G. Filippini, L. Arpaia, P. Bonneton and M. and Ricchiuto, Eur. J. Mech. B Fluids, Modeling analysis of tidal bore formation in convergent estuaries, https://doi.org/10.1016/j.euromechflu.2018.01.001.

[98] J.T. Kirby and M. Derakhti, M., 2017. Short-crested wave breaking, Eur. J. Mech. B Fluids, https://doi.org/10.1016/j.euromechflu.2017.11.001.

[99] A. Senthilkumar and H. Kalisch, Wave breaking in the KdV equation on a flow with constant vorticity, Eur. J. Mech. B Fluids, https://doi.org/10.1016/j.euromechflu.2017.12.006.

[100] A.Iafrati, F. De Vita and R.Verzicco, Effects of the wind on the breaking of modulated wave trains, Eur. J. Mech. B Fluids, https://doi.org/10.1016/j.euromechflu.2018.03.012.

[101] M.P. Buckley and F. Véron, The turbulent airflow over wind generated surface waves Eur. J. Mech. B Fluids,, https://doi.org/10.1016/j.euromechflu.2018.04.003.

[102] L. Smith J. Kolaas, A. Jensen and K. Sveen, X-ray measurements of plunging breaking solitary waves, Eur. J. Mech. B Fluids, https://doi.org/10.1016/j.euromechflu.2018.03.007.

[103] C. Lin, M.-J. Kao, W.-Y. Wong, Y.-P. Shao, C.-F. Fu, J.-M. Yuan and R. Raikar, Effect of Leading Waves on Velocity Distribution of Undular Bore Traveling over Sloping Bottom, Eur. J. Mech. B Fluids, https://doi.org/10.1016/j.euromechflu.2018.05.005

[104] P. Lubin, O. Kimmoun, F. Véron and S. Glockner, Discussion on instabilities in breaking waves: vortices, air-entrainment and droplet generation, Eur. J. Mech. B Fluids, https://doi.org/10.1016/j.euromechflu.2018.05.006

[105] A. Jensen, Solitary wave impact on a vertical wall, Eur. J. Mech. B Fluids,, https://doi.org/10.1016/j.euromechflu.2018.05.004 